\title{
Galaxies behind the deepest extinction layer of the southern Milky Way
}

\author{
Renée C. Kraan-Korteweg ${ }^{1}$, Bärbel Koribalski ${ }^{2}$, and Sebastian Juraszek ${ }^{3,2}$ \\ 1 Departamento de Astronomia, Universidad de Guanajuato, Mexico \\ kraan@norma.astro.ugto.mx \\ 2 Australia Telescope National Facility, CSIRO, P.O. Box 76, Epping, Australia \\ bkoribal@atnf.csiro.au \\ 3 School of Physics, Univ. of Sydney, NSW 2006, Australia, sjurasze@atnf.CSIRO.AU
}

\begin{abstract}
About 25\% of the optical extragalactic sky is obscured by the dust and stars of our Milky Way. Dynamically important structures might still lie hidden in this zone. Various approaches are presently being employed to uncover the galaxy distribution in this Zone of Avoidance (ZOA). Results as well as the different limitations and selection effects from these multi-wavelengths explorations are being discussed. Galaxies within the innermost part of the Milky Way - typically at a foreground obscuration in the blue of $A_{\mathrm{B}} \gtrsim 5^{m}$ and $|b| \lesssim \pm 5^{\circ}$ - remain particularly difficult to uncover except for H I-surveys: the Galaxy is fully transparent at the $21 \mathrm{~cm}$ line and H I-rich galaxies are easy to trace. We will report here on the first results from the systematic blind H I-search $\left(v \leq 12700 \mathrm{~km} \mathrm{~s}^{-1}\right)$ in the southern Zone of Avoidance which is currently being conducted with the Parkes Multibeam (MB) Receiver.
\end{abstract}

\section{Introduction}

To understand the dynamics within the local Universe - the mass distribution and the local velocity field with its peculiar and streaming motions - a detailed map of the 3-dimensional galaxy distribution is highly desirable. However, the dust extinction and confusion with stars in the disk of our Galaxy make this very difficult for $\sim 25 \%$ of the sky, and the following questions remain unanswered:

Could a nearby Andromeda-like galaxy have escaped detection to date, hence change our understanding of the internal dynamics and mass derivations of the Local Group (LG), and the present density of the Universe from timing arguments (Peebles 1994)?

Is the dipole in the Cosmic Microwave Background Radiation (direction and amplitude) entirely explained by the gravity on the LG from the irregular mass/galaxy distribution? As the nearest galaxies $\left(v<300 \mathrm{~km} \mathrm{~s}^{-1}\right)$ generate $20 \%$ of the total dipole moment (Kraan-Korteweg 1989) nearby individual galaxies are equally important as massive groups, clusters and voids.

Is the mass overdensity in the Great Attractor (GA) region - postulated from a large-scale systematic flow of galaxies towards $(\ell, b, v) \sim\left(320^{\circ}, 0^{\circ}, 4500 \mathrm{~km} \mathrm{~s}^{-1}\right)$ (Kolatt et al. 1995) - in the form of galaxies, hence does light trace mass? 
Does the Supergalactic Plane, other superclusters, walls and voids connect across the Milky Way and might other large-scale structures (LSS) have gone undetected due to this 'zone of avoidance'?

\section{Multiwavelengths explorations of the southern $\mathrm{ZOA}$}

Various approaches are presently being employed to uncover the galaxy distribution in the ZOA: deep optical searches, far-infrared (FIR), near-infrared (NIR) surveys and blind H I searches. All methods produce new results, but all suffer from (different) limitations and selection effects.

OPTICAL: Nearly the whole southern ZOA has been systematically surveyed for highly obscured but still visible galaxies using existing sky surveys ( $c f$. Woudt 1998, for a detailed overview). These surveys achieve a considerable reduction of the ZOA and have uncovered distinct LSS unrelated with the foreground extinction. Follow-up redshift observations have revealed a number of dynamically important structures such as e.g., the nearby overdensity in Puppis (Lahav et al. 1993) and the massive cluster A3627 at the core of the GA (KraanKorteweg et al. 1996, cf. Fig. 1). Deep optical surveys are not biased with respect to any particular morphological type. However, for foreground extinctions above $\mathrm{A}_{B} \gtrsim 5^{m}$ (H I-column-densities $\mathrm{N}_{\mathrm{HI}} \gtrsim 6 \cdot 10^{21} \mathrm{~cm}^{-2}$ ), the ZOA remains fully opaque ( $c f$. inner contour in Fig. 1). For the southern Milky Way this corresponds roughly to $|b| \lesssim \pm 5^{\circ}$.

FIR: The IRAS Point-Source Catalog (PSC) has been exploited in the last decade to identify galaxy candidates behind the ZOA. Using different colour selection criteria, galaxy candidates were followed up by $\mathrm{HI}$ radio surveys (e.g., Lu et al. 1990) or by inspection of plates (e.g., Takata et al. 1996). To avoid confusion with Galactic sources, K-band snapshots have proved very efficient (Saunders et al. 1994). Confirmed IRAS galaxies can be merged with IRAS galaxy samples outside the ZOA to produce uniform whole-sky samples for LSS studies. But bright spiral and starburst galaxies dominate these samples.

NIR: The recent near infrared (NIR) surveys, 2MASS (Skrutskie et al. 1997) and DENIS (Epchtein 1997), provide complementary data. NIR surveys are sensitive to early-type galaxies, are tracers of massive groups and clusters missed in IRAS and H I surveys, have little confusion with Galactic objects and are less affected by absorption than optical surveys.

In a pilot study, we examined the effeciency of uncovering galaxies at high extinctions with DENIS images ( $c f$. Schröder et al. 1997 \& Kraan-Korteweg et al. 1998): highly obscured, optically invisible galaxies can indeed be traced to lower latitudes $(|b| \gtrsim 1-1.5)$ than deep optical surveys. This is not only of interest in charting early-type galaxies but also with respect to the combination of $\mathrm{H}$ I data of heavily obscured spiral galaxies detected in blind H I surveys (cf. below) with 
NIR data, and therewith the possibility to extend the peculiar velocity field into the ZOA via the NIR Tully-Fisher relation.

H I: In the regions of highest obscuration and infrared confusion the Galaxy is fully transparent to the $21-\mathrm{cm}$ line radiation of neutral hydrogen. H I-rich galaxies can readily be found at lowest latitudes through detection of their redshifted 21-cm emission. Only low-velocity extragalactic sources (blue- and redshifted) within the strong Galactic HI emission will be missed, and - because of baseline ripple - galaxies close to radio continuum sources.

Until recently, radio receivers were not sensitive and efficient enough to attempt large systematic surveys of the ZOA. In a pilot survey with the late $300-\mathrm{ft}$ telescope of Green Bank, Kerr \& Henning (1987) surveyed 1.5\% of the ZOA and detected 16 new spiral galaxies. Since then a systematic shallow search for nearby, massive galaxies has been completed in the north (Henning et al. 1998), yielding five objects including Dwingeloo 1 (Kraan-Korteweg et al. 1994).

\section{The Parkes Multibeam Survey in the southern ZOA}

In March 1997, a systematic blind H I survey began with the Multibeam Receiver (13 beams in the focal plane array) at the $64 \mathrm{~m}$ Parkes telescope in the most opaque region of the southern Milky Way $\left(213^{\circ} \leq \ell \leq 33^{\circ} ;|b| \leq 5^{\circ}\right)$. The ZOA will be surveyed along constant Galactic latitudes in 23 contiguous fields of length $\Delta \ell=8^{\circ}$. The ultimate goal is 25 scans per field where adjacent strips will be offset in latitude by $\Delta b=1.5$ for homogeneous sampling. With a total observing time of $1500 \mathrm{~h}$, we will obtain an effective integration time of $25 \mathrm{~min} /$ beam with a $3 \sigma$ detection limit of $15 \mathrm{mJy}$. Roughly 3000 detections are predicted for the covered velocity range of $-1200 \lesssim v \lesssim 12700 \mathrm{~km} \mathrm{~s}^{-1}$ (Staveley-Smith 1997). This allows the detection of dwarfs with $\mathrm{HI}$-masses as low as $10^{6} \mathrm{M}_{\odot}$ in the local neighbourhood, and will be sensitive to normal Sc galaxies well beyond the Great Attractor region. As a byproduct, the survey will produce a high resolution integrated column density map of the southern Milky Way and a detailed catalog of high velocity clouds ( $c f$. Putnam et al. 1998).

\subsection{First Results from the Parkes Multibeam survey}

At the time of this meeting, the whole southern ZOA survey had been surveyed twice $\left(\Delta b=17^{\prime}\right.$, rms $\left.\sim 20 \mathrm{mJy}\right)$. The cubes of the Hanning smoothed data $\left(26 \mathrm{~km} \mathrm{~s}^{-1}\right.$ resolution) were inspected visually for 21 of the fields $\left(220^{\circ} \leq \ell \leq 4^{\circ}\right)$ and all galaxy candidates with H I fluxes $\gtrsim 100$ mJy were catalogued.

87 galaxies were uncovered in this way. Four were seen in more than one cube. Though galaxies up to $6500 \mathrm{~km} \mathrm{~s}^{-1}$ were identified, most of the galaxies $(80 \%)$ are quite local $\left(\mathrm{v}<3500 \mathrm{~km} \mathrm{~s}^{-1}\right)$ due to the (yet) low sensitivity. In the low-extinction Puppis region ( $c f$. Fig. 1), a large fraction of the galaxies and their velocities were already known. In the remaining ZOA, about $1 / 3$ have a counterpart in NED or the deep optical surveys. 


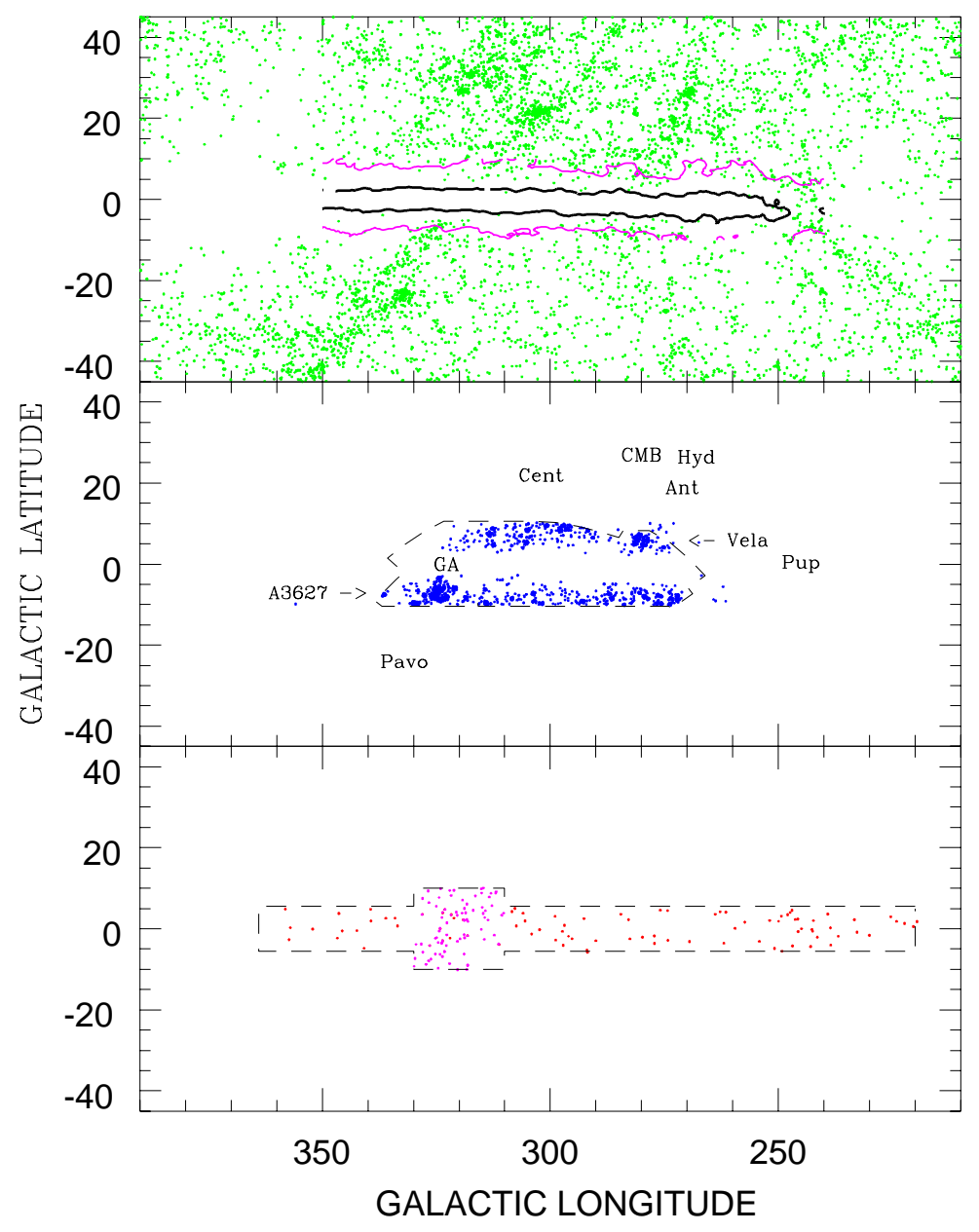

Fig. 1. Galaxies with $\mathrm{v}<10000 \mathrm{~km} \mathrm{~s}^{-1}$. Top panel: literature values (LEDA), superimposed are extinction levels $\mathrm{A}_{B} \sim 11^{\mathrm{m}} 5$ and $5^{\mathrm{m}}$; middle panel: follow-up redshifts (ESO, SAAO and Parkes) from deep optical ZOA survey with locations of clusters and dynamically important structures; bottom panel: redshifts from shallow MB-ZOA and deeper GA survey in H I with the Parkes radio telescope.

The distribution of the H I-detected galaxies is shown in the lower panel of Fig. 1. Here we also display the results by Juraszek et al. (in prep.) in the GA region. In this high priority area, defined as $310^{\circ} \leq \ell \leq 330^{\circ},|b| \leq 10^{\circ}$, four scans rms $(\sim 15 \mathrm{mJy})$ were analyzed and 82 galaxies charted. This area hence probes deeper and finds - not unexpectedly - a peak in the velocity distribution between 3000 and $4500 \mathrm{~km} \mathrm{~s}^{-1}$ in the GA direction. The top panel of Fig. 1 shows the distribution of all galaxies with velocities $\mathrm{v} \leq 10000 \mathrm{~km} \mathrm{~s}^{-1}$ centered 


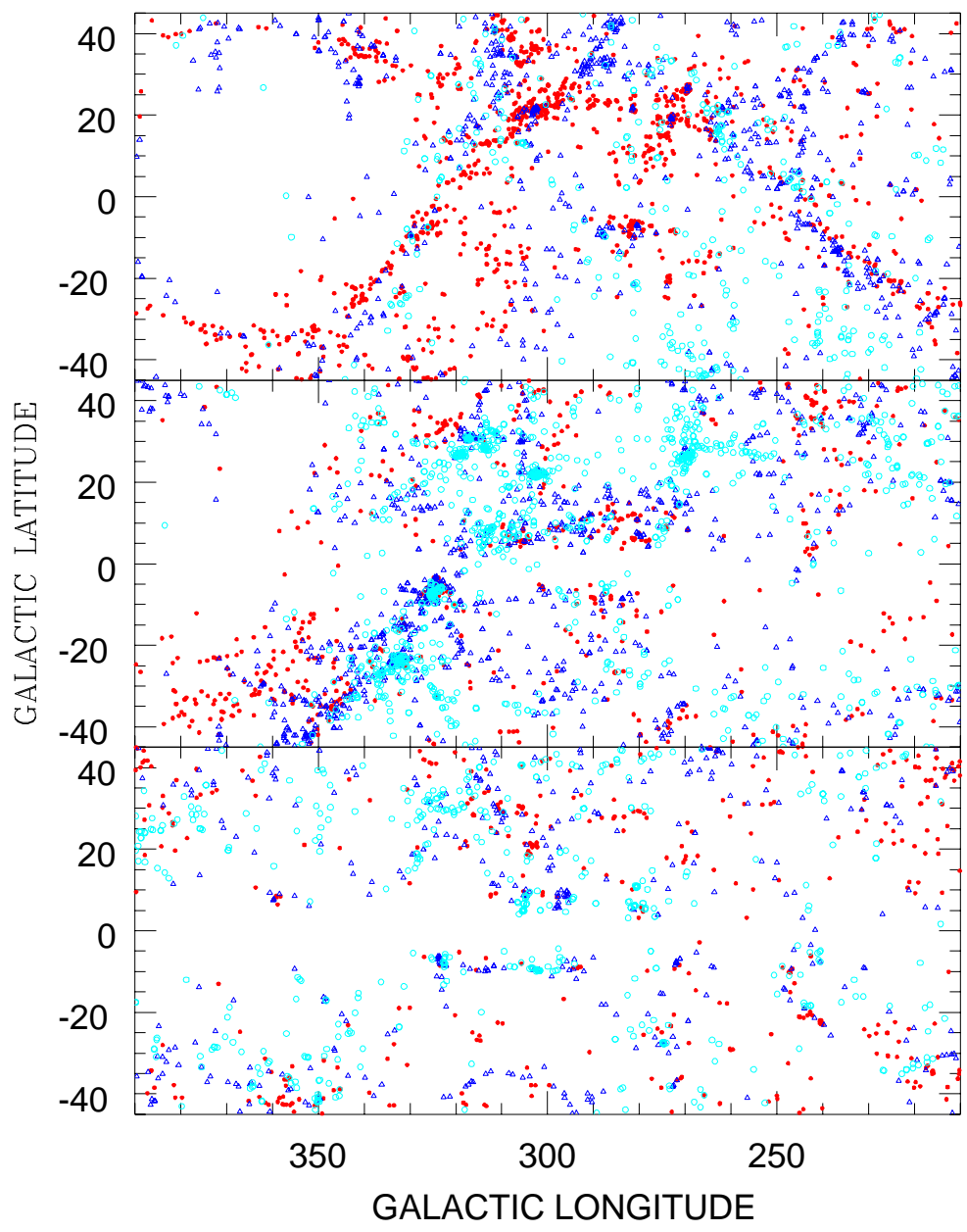

Fig. 2. Redshift slices from data in Fig. 1: $500<\mathrm{v}<3500$ (top), $3500<\mathrm{v}<6500$ (middle), $6500<\mathrm{v}<9500 \mathrm{~km} \mathrm{~s}^{-1}$ (bottom). The open circles mark the nearest $\Delta \mathrm{v}=1000 \mathrm{~km} \mathrm{~s}^{-1}$ slice in a panel, then triangles, then the filled dots the 2 more distant ones.

on the southern Milky Way. Note the near full lack of galaxy data for extinction levels $\mathrm{A}_{B} \sim 1 \mathrm{~m} 5$ (outer contour). The middle panel results from the follow-up observations of the optical galaxy search by Kraan-Korteweg and collaborators. Various new overdensities become apparent at low latitudes. But the innermost part of our Galaxy remains obscured $\left(\mathrm{A}_{B} \gtrsim 5^{m},|b| \lesssim 5^{\circ}\right)$. Here, the blind H I data ( $c f$. lower panel) finally can provide the missing link for LSS studies.

In Fig. 2, the data of Fig. 1 are combined in redshift slices. The achieved sensitivity in the current MB HI-survey fills in structures all the way across the ZOA for the upper panel $\left(\mathrm{v}<3500 \mathrm{~km} \mathrm{~s}^{-1}\right)$ for the first time. Note the 
continuity of the thin filamentary sine-wave-like structure that dominates the whole southern sky, and the prominence of the Local Void. This feature is very different from the thick, foamy Great Wall-like structure, the GA, in the middle panel. With the full sensitivity aimed at with the MB-survey, we will be able to fill in the LSS in the more distant panels of Fig. 2 as well.

ATCA follow-up observations of three very extended $\left(20^{\prime}\right.$ to $\left.\gtrsim 1^{\circ}\right)$, nearby $\left(\mathrm{v}<1500 \mathrm{~km} \mathrm{~s}^{-1}\right)$ sources revealed them to be interesting galaxies/complexes, with unprecedented low H I column densities (cf. Staveley-Smith et al. 1998).

\section{Conclusions}

The combination of the complementary multiwavelength surveys allow a new probing of LSS in the 'former' ZOA. The H I surveys are particularly powerful at the lowest latitudes. But future merging of ZOA data with catalogs outside the ZOA will have to be done with care to obtain 'unbiased' whole-sky surveys.

From the sensitivity attained with the first 2 scans of the ZOA MB-survey it can be maintained that no Andromeda or other H I-rich Circinus galaxy is lurking undetected behind the extinction layer of the southern Milky Way.

Acknowledgements - The help of the HIPASS ZOA team members R.D. Ekers, A.J. Green, R.F. Haynes, P.A. Henning, R.M. Price, E. Sadler, and L. Staveley-Smith is gratefully acknowledged.

\section{References}

Epchtein, N. (1997): The Impact of Large Scale Near-Infrared Surveys eds. F. Garzon et al. (Kluwer: Dordrecht) p. 15

Henning, P.A., Kraan-Korteweg, R.C., Rivers, A.J., Loan, A.J., Lahav, O., Burton, W.B (1998): AJ 115, 584

Kerr, F.J., Henning, P.A. (1987): ApJ 320, L99

Kolatt, T., Dekel, A., Lahav, O. (1995), MNRAS 275, 797

Kraan-Korteweg, R.C. (1989): Rev. in Modern Astron. 2, ed. G. Klare (Springer: Berlin), p119

Kraan-Korteweg R.C., Loan A.J., Burton W.B., Lahav O., Ferguson H.C., Henning P.A., Lynden-Bell D. (1994): Nat 372, 77

Kraan-Korteweg, R.C., Woudt, P.A., Cayatte, V., Fairall, A.P., Balkowski, C., Henning, P.A. (1996): Nat 379, 519

Kraan-Korteweg, R.C., Schröder, A., Mamon, G., Ruphy, S. (1998): The Impact of Near-Infrared Surveys on Galactic and Extragalactic Astronomy, ed. N. Epchtein (Kluwer: Dordrecht), in press (astro-ph/9711226)

Lahav, O., Yamada, T., Scharf, C.A. Kraan-Korteweg, R.C. (1993): MNRAS 262, 711

Lu, N.Y., Dow, M.W., Houck, J.R., Salpeter, E.E., Lewis, B.M. (1990): ApJ 357, 388

Peebles, P.J.E. (1994): ApJ 429, 43

Putnam, M.E. et al. (1998): Nat, submitted

Saunders, W. et al. (1994): Unveiling Large-Scale Structures Behind the Milky Way, eds. C. Balkowski, R.C. Kraan-Korteweg, ASP Conf. Ser. 67, 257 
Schröder, A., Kraan-Korteweg, R.C., Mamon, G.A., Ruphy, S. (1997): 'Extragalactic Astronomy in the Infrared, eds. Trinh Thuân et al. (Frontières: Gif-sur-Yvette), p381 Staveley-Smith, L. (1997): PASA 14, 111

Staveley-Smith, L., Juraszek, S., Koribalski, B.S., Ekers, R.D., Green, A.J., Haynes, R.F., Henning, P.A., Kesteven, M.J., Kraan-Korteweg, R.C., Price, R.M., Sadler, E.M. (1998): AJ, submitted

Skrutskie, M.F., et al. (1997): The Impact of Large Scale Near-Infrared Surveys, eds. F. Garzon et al. (Kluwer: Dordrecht) p. 25

Takata, T., Yamada, T., Saito, M. (1996): ApJ 457, 693

Woudt, P.A. (1998): Ph.D. thesis, Univ. of Cape Town. 